Results: Data from 23 patients were analysed during the 52 weeks observational period. Mean age was 54.7 years, $60 \%$ of the patients were female. Mean DAS28 at baseline was 4.26, mean BSA 9\%, whereas mean values for SJC were 5.9 and TJC 11.4 using 66/68 joint count. All patients were negative for ACPA and rheumatoid factor

ROC analysis revealed that a DACT cut-off of 4.55 at baseline, indicating moderate expression of fluorescence intensities in context of disease activity, shows a predictive quality to LDA achievement at W52 with $80 \%$ specificity, $78 \%$ sensitivity and likelihood ratios of 3.89 (LQ+) and 0.28 (LQ-). The corresponding AUC value is $0.717(95 \% \mathrm{Cl}=[0.393,1] ; \mathrm{p}=0.146)$. Compared to clinical disease measurements such as baseline DAS28, TJC or SJC, the DACT at BL is more discriminative to identify patients who attain LDA at W52.

Conclusions: This interim analysis of the XPLORE study shows promising data for the use of FOI as possible imaging biomarker for disease activity measure and prediction of response in PSA-patients newly treated with antiTNF-therapy: Baseline values evaluated using the automated computer-based reading of the fluorescence intensities with a cut-off of 4.55 are predictive for later achievement of DAS28 low-disease activity or remission within the treatment course. Data will be verified in a larger cohort of the XPLORE study.

Disclosure of Interest: M. Köhm Grant/research support from: Pfizer Germany, S. Ohrndorf: None declared, T. Rossmanith: None declared, A. Foldenauer: None declared, U. Henkemeier: None declared, G. Schmittat: None declared, J. Berger Employee of: Xiralit GmbH, H. Burkhardt Grant/research support from: Pfizer Germany, F. Behrens Grant/research support from: Pfizer Germany

DOI: 10.1136/annrheumdis-2018-eular.6764

\section{AB0881 ASSOCIATION OF RS12218 POLYMORPHISM IN SAA1GENE WITH LUMBAR SPINE SYNDESMOPHYTES IN THE RUSSIAN ANKYLOSING SPONDYLITISPOPULATION. A PILOT STUDY}

M. Krylov ${ }^{1}$, S. Erdes ${ }^{1}$, A. Starkova ${ }^{1}$, E. Samarkina ${ }^{1}$, I. Guseva ${ }^{1}$, N. Konovalova ${ }^{2}$ D. Varlamov ${ }^{2} .{ }^{1}$ V.A. Nasonova Research Institute of Rheumatology, ${ }^{2}$ All-Russian Research Institute of Agricultural Biotechnology, Moscow, Russian Federation

Background: Ankylosing spondylitis (AS) is a chronic inflammatory disease from the group of spondyloarthritis (SPa). Earlier studies showed a correlation between SAA1 gene polymorphism, encoding serum amyloid $A$, and the development of secondary AA-amyloidosis in familial Mediterranean fever and rheumatoid arthritis in Caucasian and Asian populations. The Moriguchi et al (2005) study showed that the $-13 \mathrm{~T} / \mathrm{C}$ polymorphism in the gene 5'-flanking region ( $r$ 12218) is a better marker of AA-amyloidosis than mapping of polymorphisms in SAA1 exon 3 (SAA1.1 and SAA1 1.3). Data on rs12218 polymorphism contribution into predisposition to AS and its clinical phenotypes are very scarce. One of the clinical phenotypes, determining the severity of spine damage, is associated with presence of syndesmofytes (SM) in the lumbar (SMI), thoracic (SMt) and cervical (SMc) spine, confirmed by $\mathrm{x}$-ray data.

Objectives: To study potential associations of rs12218 polymorphism in SAA1 gene with AS and phenotypes of radiographic progression, with the presence of SMI, SMt and SMc, and correlation with BASDAI, BASFI and ASAS indices.

Methods: rs 12218 polymorphism was studied in 112 subjects: 47 AS patients (37 males and 10 females, mean age 40y, mean disease duration 213 weeks, mean age at onset 22y, positive for HLA-B27), and 65 healthy volunteers (controls). Genotyping was performed using allele-specific polymerase chain reaction in real time (PCR-RT).

Results: The Pearson Correlation analysis showed negative correlation between rs12218 polymorphism and presence of SMI, as well as BASDAI, BASFI scores $(r=-0.39, r=-0.35$ and $r=-0.36, p=0.006, p=0.017, p=0.014$, respectively). There were no correlations between rs12218 and pts' age, AS duration and pts' age at AS onset. There were similar rs12218 allele distribution rates between AS patients and the controls. rs $12218 \mathrm{C}$ allele rates were significantly higher in SMl group $(n=23)$ compared to subjects without SMl $(n=24)[50.0 \%$ vs. $15.2 \%$, $\mathrm{p}=0.001]$. No association was established between $\mathrm{C}$ allele and presence of SM in other parts of the spine. A correlation between BASDAI and BASFI scores and SAA1 gene rs12218 polymorphism was established. Mean BASDAI score was significantly higher in carriers of TC and CC genotypes compared to carriers of TT genotype $(5.6 \pm 1.3$ vs. $3.9 \pm 2.3, p=0.004)$. The mean BASFI scores in carriers of the respective genotypes were $(6.1 \pm 2.3$ vs $4.1 \pm 2.8, p=0.012)$. No significant correlation was found between rs12218genotypes and mean ASDAS score values. Conclusions: Therefore, this pilot study is the first to show the possible participation of rs12218 polymorphism in SAA1 gene in AS pathogenesis in Russian population. We suggest that $C$ allele may be is a risk factor predisposing to SMI [OR $5,1495 \% \mathrm{Cl}(1.75-16.17), \mathrm{p}=0.001]$. The data obtained on a limited sample of patients require further validation on larger samples of patients involving different population groups.

Disclosure of Interest: None declared

DOI: 10.1136/annrheumdis-2018-eular.2466

\section{AB0882 \\ ACHILLES ENTHESITIS IN THE PATIENTS WITH SPONDYLOARTHRITIS: RELATIONSHIP WITH MUSCLE STRENGTH, ACTIVITIES OF DAILY LIVING AND QUALITY OF LIFE}

Ö. Kuru, B. Farisoğulları, G. Alayli, A. Bilgici, D. Durmuş. Physical Medicine and Rehabilitation, Ondokuz Mayıs University Medical Faculty, Samsun, Turkey

Background: Enthesitis is a central feature of spondyloarthritis (SpA). In SpA the entheses of the lower extremities are more commonly involved than those of the upper limbs, and the heel is the most frequent site. Investigation of peripheral enthesitis in SpA is based on clinical findings and/or imaging findings. The involvement of Achilles tendon may lead to pain, movement restrictions, decrease in muscle strength, and eventually a diminished quality of life (QoL).

Objectives: In the present study, we aimed to evaluate clinical enthesopathy and relation with ankle muscle strength, activities of daily living (ADLs) and foot and ankle related QoL in the patients with $\mathrm{SpA}$.

Methods: Sixty SpA patients fulfilling the Assessment of SpondyloArthritis International Society (ASAS) classification criteria for SpA (M/F=39/21) $(35.61 \pm 9.85$ years) and 50 healthy controls $(M / F=32 / 18)(35.40 \pm 10.62$ years) were enrolled in the study. Clinical enthesopathy was defined by the presence of at least one of the spontaneous pain, tenderness elicited by pressure, mobilisation and contraction against resistance of the corresponding tendons and local swelling of the enthesis. Pain by visual analogue scale (VAS), disease activity by Bath Ankylosing Spondylitis Disease Activity Index (BASDAI), functional capacity by Bath Ankylosing Spondylitis Functional Index (BASFI), enthesitis severity by SPARCC index was assessed in the patients. Isokinetic measurements of ankle dorsiflexion and plantarflexion were performed by the isokinetic dynamometer. The participants were tested at $30^{\circ}$ and $120^{\circ} / \mathrm{sec}$ angular velocities. Pain, other symptoms (stiffness, swelling, range of motion), ADLs, sport and recreational activities, and foot and ankle-related QoL were evaluated by the Foot and Ankle Outcome Score (FAOS) in which higher scores indicate lesser problems and/or functional limitations.

Results: There was no significant difference between the patients and controls regarding age, sex, and body mass index. Clinical enthesopathy was detected in $36.7 \%$ of the SpA patients. Although ankle plantarflexion and dorsiflexion muscle strength in all angular velocities were lower in the SpA patients, the difference did not reach statistical significance $(p>0.05)$. All of the FAOS subscales were found to be significantly lower in the patients with SpA than in the controls $(p<0.001)$ When the SpA patients were divided into two groups as with clinical enthesopathy $(n=22)$ and without clinical enthesopathy $(n=38)$, there were significant differences between the groups regarding VAS pain, BASDAI, BASFI and SPARCC scores whereas there was no significant difference in muscle strength. Also, all FAOS subscale scores were significantly lower in the patients with clinical enthesopathy. Additionally, in the patients with $\mathrm{SpA}$, while there were negative correlations between VAS pain, BASDAI, BASFI, SPARCC and FAOS subscale scores, there was a positive correlation between ankle muscle strength and FAOS scores $(\mathrm{p}<0.05)$.

Conclusions: We found that all the FAOS subscale scores were lower in the SpA patients and they were correlated with clinical findings. The results of our study indicate that even though there was not a significant decrease in the muscle strength, ADLs, sport and recreation activities, foot-related QoL are poorly affected in the SpA patients with Achill enthesitis.

Disclosure of Interest: None declared

DOI: 10.1136/annrheumdis-2018-eular.6361

\section{$\mathrm{AB} 0883$ \\ ASSESSMENT OF EARLY MYOCARDIAL DYSFUNCTION USING SPECKLE TRACKING ECHOCARDIOGRAPHY IN PATIENTS WITH RADIOGRAPHIC AND NONRADIOGRAPHIC AXIAL SPONDYLOARTHRITIS}

V. Emren ${ }^{1}, \underline{\text { O. Gercik }}{ }^{2}$, E. Ozdemir ${ }^{1}$, D. Solmaz ${ }^{2}$, S. Gucenmez ${ }^{2}$, N. Eren ${ }^{1}$,

M. Tokac ${ }^{1}$, G. Kabadayi ${ }^{2}$, S. Akar ${ }^{2}{ }^{1}$ Cardiology; ${ }^{2}$ Rheumatology, Izmir Katip Celebi University School of Medicine, Izmir, Turkey

Background: Axial spondyloarthritis (axSpA) is a chronic inflammatory disease that mainly affects axial skeleton. Although some differences like sex and objective signs of inflammation were described between these two subgroups, overall disease burden was found to be similar in radiographic ( $r$-) and non-radiographic (nr-) axSPA patients. The association of chronic inflammation with cardiac dys function was well documented in many inflammatory rheumatic diseases. However it was not assessed in the subgroups of axSpA patients. Advanced twodimensional (2D) speckle tracking echocardiographic analysis is more sensitive and accurate method of early detection of myocardial dysfunction than the conventional 2D transthoracic echocardiography (TTE).

Objectives: To evaluate the left ventricular function by using speckle tracking echocardiography in patients with both $r$ - and nr-axSpA.

Methods: In total 64 patients with r-axSpA (70\% male) and age- and sexmatched 27 patients with nr-axSpA (63\% male) and 30 healthy control subjects 